\title{
Role of $c$-kit in mammalian spermatogenesis
}

P. Rossi, C. Sette, S. Dolci, and R. Geremia

Dipartimento di Sanità Pubblica e Biologia Cellulare, Sezione di Anatomia, Università "Tor Vergata", Roma, Italy

Reprinted from:

\section{JOURNAL OF \\ ENDOCRINOLOGICAL INVESTIGATION}

An International Journal

The Official Journal of the Italian Society of Endocrinology 


\title{
Role of c-kit in mammalian spermatogenesis
}

\author{
P. Rossi, C. Sette, S. Dolci, and R. Geremia
} Dipartimento di Sanità Pubblica e Biologia Cellulare, Sezione di Anatomia, Università "Tor Vergata",
Roma, Italy

\begin{abstract}
The tyrosine-kinase receptor c-kit and its ligand, stem cell factor (SCF), are essential for the maintenance of primordial germ cells (PGCs) in both sexes. However, c-kit and a postmeiotic-specific alternative c-kit gene product play important roles also during post-natal stages of spermatogenesis. In the adult testis, the c-kit receptor is re-expressed in differentiating spermatogonia, but not in spermatogonial stem cells, whereas SCF is expressed by Sertoli cells under FSH stimulation. SCF stimulates DNA synthesis in type A spermatogonia cultured in vitro, and injection of anti-c-kit antibodies blocks their proliferation in vivo. A point mutation in the c-kit gene, which impairs SCF-mediated activation of phosphatydilinositol 3-kinase, does not cause any
\end{abstract}

\section{ROLE OF C-KIT IN THE EMBRYONAL STAGES OF GAMETOGENESIS}

The proto-oncogene $c$-kit was initially identified as the cellular homolog of the viral oncogene $v$-kit (1). The main product of the c-kit gene is a $\sim 150 \mathrm{kDa}$ tyrosine-kinase receptor (Fig. 1) that belongs to the same family as the $\alpha$ and $\beta$ PDGF receptors and the CSF-1 receptor $(2,3)$. A series of studies led to the identification of $c-k i t$ as the product of the W (dominant White spotting) locus $(4,5)$. Mutations in this locus in mice cause defects in pigmentation, anemia and sterility, due to the lack of progenitor cells belonging to the melanocytic, hematopoietic and germ cell lineages, and very similar defects are associated with mutations in the SI (Steel) locus (6). Although $W$ and $S I$ mice show the same phenotype genetic studies suggested that the defect carried by the $W$ mutations is intrinsic to the progenitor cells of the three affected lineages, whereas the defects carried by the SI mutations derives from the environment surrounding the progenitor cells (6).

\footnotetext{
Key-words: C-kit, stem cell factor, spermatogenesis, fertilization.

Correspondence to: Prof. Pellegrino Rossi, Dipartimento di Sanità Pubblica e Biologia Cellulare, Sezione di Anatomia, Università "Tor Vergata", Via O. Raimondo 8, 00173 Roma, Italy.

E-mail: pellegrino.rossi@med.uniroma2.it
}

significant reduction in PGCs number during embryonic development, nor in spermatogonial stem cell populations. However males are completely sterile due to a block in the initial stages of spermatogenesis, associated to abolishment of DNA-synthesis in differentiating $A_{1}-A_{4}$ spermatogonia. With the onset of meiosis c-kit expression ceases, but a truncated c-kit product, tr-kit, is specifically expressed in post-meiotic stages of spermatogenesis, and is accumulated in mature spermatozoa. Microinjection of tr-kit into mouse eggs causes their parthenogenetic activation, suggesting that it might play a role in the final function of the gametes, fertilization. (J. Endocrinol. Invest. 23: 609-615, 2000) ${ }^{\odot}$ 2000, Editrice Kurtis

The parallelism between the $W$ and SI phenotype led to the hypothesis the SI locus contained the gene encoding the growth factor specifically recognized by the c-kit receptor. This hypothesis was indeed confirmed with the cloning of the CDNA of a pleiotropic growth factor in the SI locus (7-9) which was able to bind and activate $c-k i t$, which originally received different names (MGF: mast cell growth factor; SLF: steel factor; KL: kit ligand) and is actually known as SCF (stem cell factor). SCF is produced in a soluble and a membrane bound form, resulting from alternative splicing around the exon 6 of the gene (10).

In the embryonal gonad the c-kit tyrosine kinase receptor and its ligand SCF are required for the survival and proliferation of primordial germ cells (PGCs). Indeed, homozygous $W$ and SI mice have a drastically reduced number of PGCs in the gonadal ridges (6). Several lines of evidence from in vitro studies support the hypothesis that alterations of the SCF/c-kit system are responsible for these defects in vivo. First, while c-kit is expressed by the PGCs during their migration toward the gonadal ridges, in situ hybridization and immunohistochemical experiments have shown that SCF is expressed by the cells present along the migratory path of PGCs (11). Moreover, PGCs are able to survive and proliferate in vitro in coculture with nurse cells that produce SCF, and the transmembrane form of the growth factor is much 


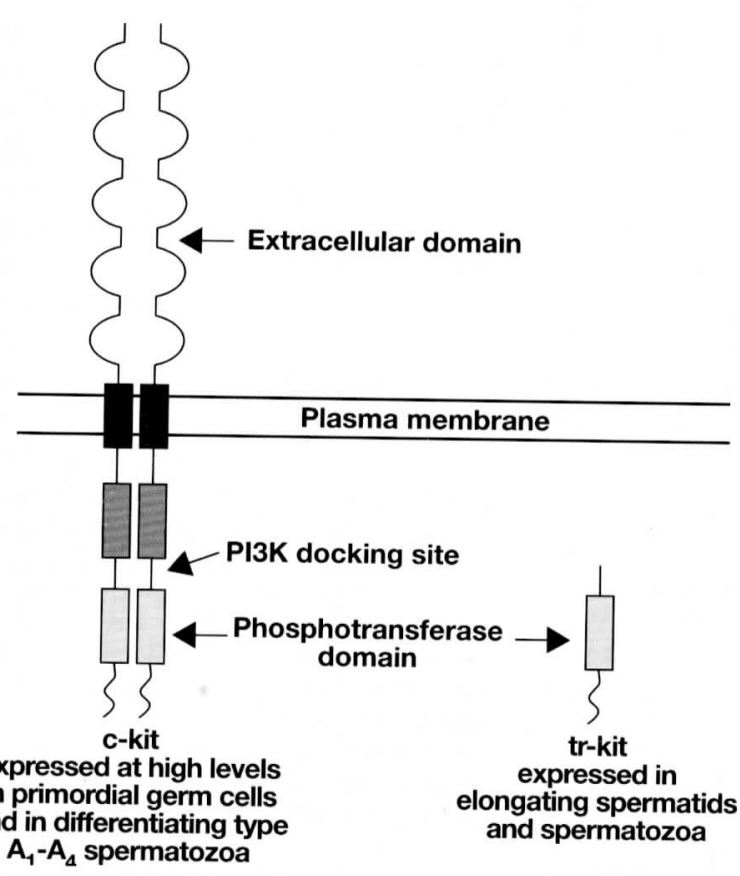

Fig. 1 - Schematic representation of two distinct c-kit gene products: the $\sim 150 \mathrm{kDa}$ transmembrane tyrosine kinase receptor expressed in both PGCs and differentiating post-natal spermatogonia (c-kit), and the $30 \mathrm{kDa}$ truncated protein expressed only in post-meiotic male germ cells (tr-kit).

more efficient than the soluble form (12-14). Furthermore, in the absence of nurse cells, PGCs undergo an apoptotic process in vitro, which can be synergistically prevented or attenuated by the presence of SCF in the culture medium (15). The end of the proliferative process of PGCs coincides with the end of c-kit expression on these cells (16). However, the role the SCF/c-kit system in gametogenesis is not limited to the embryonal period, since more recent experiments have shown that it plays a fundamental role also during post-natal stages of spermatogenesis by supporting the production and survival of mature germ cells (Fig. 2).

\section{EARLY STUDIES SUGGESTING AN IMPORTANT ROLE OF C-KIT ALSO IN POST-NATAL STAGES OF SPERMATOGENESIS}

In the adult mouse testis, the $c$-kit receptor is mainly expressed by spermatogonia $(17,18)$ and by Leydig cells (19). Type A spermatogonia are the cell type in which maximal levels of both c-kit RNA and protein are observed, followed by type B spermatogonia $(17,18,20)$. Indeed, the full length $c$-kit receptor kinase is also expressed in rat (21), mouse $(22)$, human $(23,24)$ hamster and monkey sper- matogonia (25). Expression of the c-kit receptor kinase in male mitotic germ cells starts around day 6 post-partum, when type $A_{0}$ spermatogonia differentiate into $A_{1}$ spermatogonia (17), and recent observation confirms that it is selectively expressed in differentiating type $A_{1}-A_{4}$ and type $B$ spermatogonia but not in spermatogonial stem cells $(26)$. The expression of the mRNA for the full length c-kit receptor has also been demonstrated in mouse spermatocytes, albeit at lower levels, but it ceases completely during meiosis and it is absent in post-meiotic cells $(18,27)$. Indeed, in spermatocytes the c-kit protein either is not present $(17,20,25)$ or, at least, is barely detectable (28).

The expression of $c$-kit in the only proliferating cells during spermatogenesis, the spermatogonia, has led to the hypothesis that the SCF/c-kit interaction is required for the proliferation and/or survival of these cells. Further support to this hypothesis comes from the observation that, in mice, both the soluble and membrane-bound form of SCF are expressed by the nurse cells of spermatogonia, the Sertoli cells (29-33), whose intimate contact with the germ cells is necessary for their survival and differentiation. The selective site of testicular SCF expression in the Sertoli cell has also been confirmed in the rat (34) and in humans (24). The expression of the mRNA for SCF is induced by the pituitary hormone FSH (follicle stimulating hormone) in pre-puberal mouse Sertoli cells cultured in vitro, through an increase in cAMP levels $(29,30)$. Stage-dependent induction of SCF mRNA expression by FSH has also been observed in the adult rat testis (35), and the maximal levels of SCF mRNA induction are observed in stages of the seminiferous epithelium which show the maximal sensitivity to FSH stimulation, and in which type A spermatogonia are actively dividing. Interestingly, the soluble and membrane forms of SCF are differentially expressed during testis development. Sertoli cells from pre-puberal mice mainly express the mRNA encoding for the transmembrane form, while the mRNA encoding for the soluble form is expressed at higher levels later, in coincidence with the beginning of the spermatogenic process, and the two transcripts are expressed at equivalent levels in the adult testis (30). Moreover, FSH and/or CAMP analogs, beside increasing SCF mRNA levels, also modify the splicing pattern of the two isoforms in cultured mouse Sertoli cells in favour of the mRNA encoding for the soluble form (30). In agreement with these observations is the finding that the highest levels of the transmembrane form of SCF are detected immunohistochemically in stages VII-VIII of the mouse seminiferous epithelium (28). The equivalent stages 

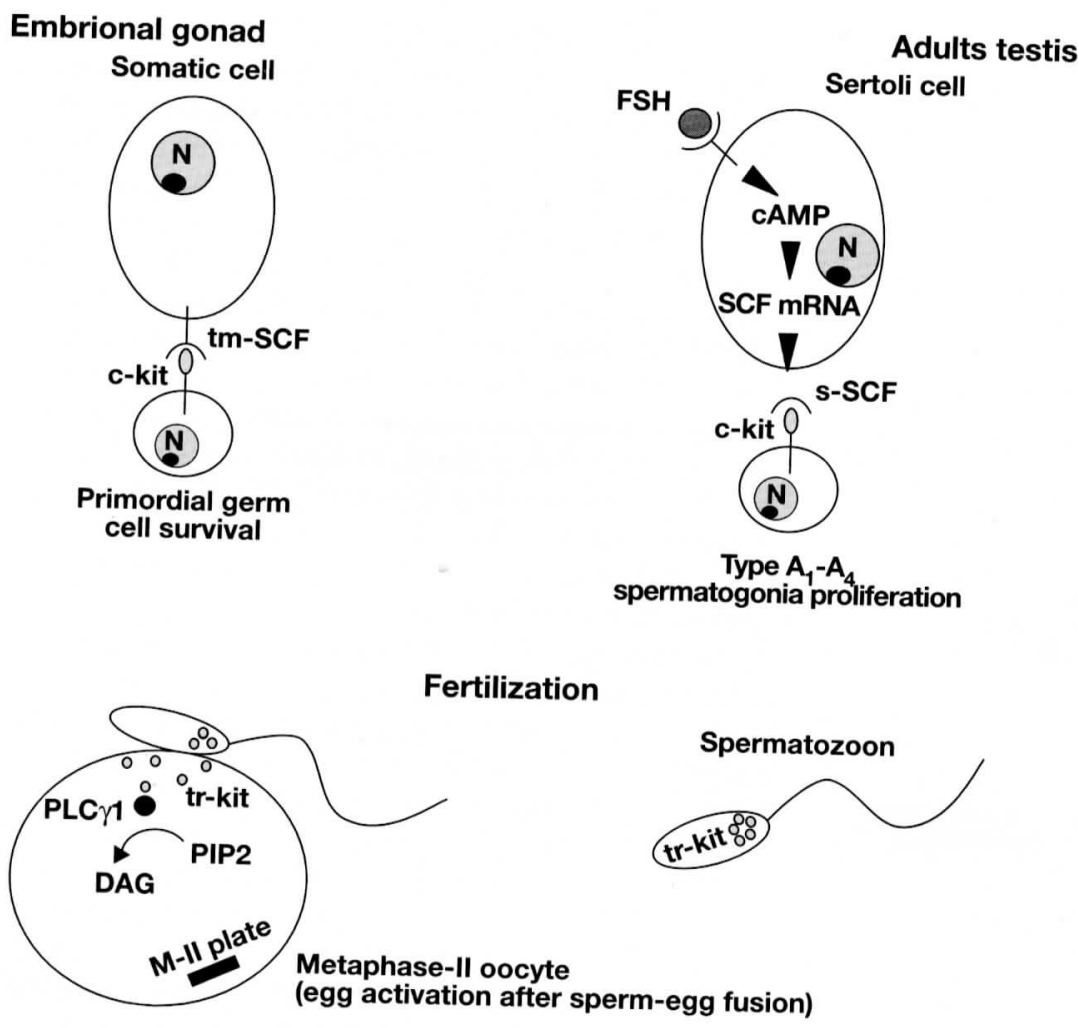

Fig. 2 - The two distinct c-kit gene products depicted in Figure 1 play different roles during gametogenesis. Activation of the full-length c-kit receptor by the transmembrane SCF (tm-SCF) is essential for $P G C$ survival in the embryonal gonad of both sexes, whereas soluble SCF (s-SCF) produced by Sertoli cells under FSH stimulation promotes proliferation of c-kit expressing differentiating spermatogonia in the adult testis. After sperm-egg fusion, transfer of sperm-carried tr-kit into metaphase II-arrested oocytes triggers egg activation at fertilization. N: nucleus; M-II plate: chromosome aligned on the metaphase-ll plate of the egg before the sperm-induced second meiotic division. in rat testis are the less sensitive to FSH stimulation in the adult testis (36). Recently, it has been reported that the splicing pattern of SCF mRNA in Sertoli cells might also be influenced by local changes of $\mathrm{pH}$ in the seminiferous epithelium (37). Early studies performed in $W$ mutant mice suggested that c-kit plays a critical role in the differentiation from type A to type B spermatogonia (38). Moreover, a peculiar Steel mutation, $S^{17 H}$, resulting in a splicing defect in the SCF cytoplasmic tail, in the homozygous condition induces sterility in males but not females, due to loss of spermatogonia during post-natal development (39).

The first evidence that $c-k i t$ plays an important role in mitotically dividing male germ cells came from the observation that addition of the soluble form of $\mathrm{SCF}$ to in vitro cultured male germ cells from 7-8 day old mice at mitotic stages of differentiation stimulates DNA synthesis selectively in type A, but not in type B spermatogonia (30). Selective induction of DNA synthesis in type A spermatogonia by soluble SCF has been confirmed using an in vitro tissue culture system for stage-defined seminiferous tubules of the adult rat testis (40). The important role of the SCF/c-kit system in the maintenance of the germ cell line in the mouse post-natal testis is also sug- gested by in vivo and in vitro experiments in which the interaction between SCF and c-kit was blocked by an antibody directed against the extracellular region of the receptor. Under these conditions, c-kit expressing type A spermatogonia, but not c-kit negative spermatogonial stem cells, are depleted (20) and unable to proliferate (41), and differentiating spermatogonia show increased levels of apoptosis (42). The role of soluble SCF in promoting survival and/or proliferation of rat and porcine spermatogonia has also been reported (43-45).

SCF has been proposed to act as a survival factor that prevents programmed cell death in cells which are intrinsically committed to proliferate, rather than a mitogenic factor which directly stimulates DNA synthesis in spermatogonia $(40,42,43)$, but accurate studies on the effect of SCF on spermatogonial cell cycle and apoptosis have not been described up to now. However, it should be reminded that appearance of actively proliferating type $A_{1}-A_{4}$ spermatogonia coincides with re-expression of the $c-k i t$ receptor, which is not expressed in post-natal spermatogonial stem cells, i.e. type $A_{0}$ spermatogonia $(17,26,41,46)$. Studies performed in cryptorchid testes of mice heterozygous for the Steel Dickie mutation, which only express a soluble, but not a trans- 
membrane form of SCF have shown that proliferation of type $A_{1}-A_{4}$ spermatogonia was apparently unaffected in surgically reversed cryptorchid testes of these animals, but a dramatic reduction in type B spermatogonia was observed, with consequent impairment of spermatogenesis (47), suggesting that soluble SCF is sufficient for divisions of type $A_{1}-A_{4}$ spermatogonia, whereas the transmembrane form of SCF is essential for preventing apoptosis of type B spermatogonia before they differentiate to meiotic stages. This would give a rationale explanation for the recent report that the transmembrane, but not the soluble form of SCF might play a role in transmeiotic progression in culture of mouse germ cells cocultured with the SCF-expressing 15-P1 cell line (28). The fact that SCF can be both a mitogenic and an anti-apoptotic stimulus in the same cell type has been clearly demonstrated in c-kit expressing cells of the hematopoietic lineage $(48,49)$.

\section{EVIDENCE FOR AN ESSENTIAL ROLE OF C-KIT IN PROLIFERATION OF DIFFERENTIATING SPERMATOGONIA}

Three very recent reports highlight the essential role played by c-kit during the spermatogenic process in the adult life. Two groups independently reported the dramatic and selective impairment of spermatogenesis in genetically modified mice in which the same discrete point mutation was introduced in the c-kit gene using a knock-in strategy $(50,51)$. Homozygous mice were generated in which the codon encoding for tyrosine 719 was mutated to phenylalanine (Y719F). The Y719 residue, after SCF-induced dimerization and autophosphorylation, is a docking site for the p85 subunit of PI3K (52), which had been previously shown to be essential for transduction of both proliferative and anti-apoptotic signals in cultured c-kit expressing cells $(48,53)$. Unexpectedly, Y719F mutant mice did not show any significant pigmentation or hematopoietic defects, and no reduction in PGC number during embryonic development. However, the mutation induced a sex- and tissue-specific defect in post-natal gametogenesis. Whereas males are completely sterile due to a block in the initial stages of spermatogenesis, females were found to be either fully fertile (50) or sub-fertile (51). Thus, a discrete point mutation in the c-kit gene, which impairs ckit mediated PI3K activation without affecting other c-kit activated signal transduction pathways, leads to a selective impairment of spermatogenesis. An accurate study of spermatogenesis in these mutant mice showed that there was no reduction in spermatogonial stem cell populations present at 6 days of post-natal age, which do not express the ckit receptor (51). Germ cell number also appeared normal at 8 days of age, when c-kit expressing differentiating $A_{1}-A_{4}$ spermatogonia are present. However BrDU incorporation studies showed a complete block of DNA-synthesis in c-kit positive spermatogonia at this age, whereas no signs of apoptosis were evident by TUNEL staining (50). Analysis at 10 days of age, when type $B$ spermatogonia and early meiotic spermatocytes should be present, showed subsequent massive reduction of germ cell number and signs of apoptotic degeneration, with no further progression of spermatogenesis; no apparent effect was observed in other c-kit expressing testicular cells, namely in Leydig cells $(50,51)$. These data clearly show that $c-k i t$ signaling through the p85 subunit of PI3K plays an essential role initially for proliferation of differentiating $c$-kit expressing type $A_{1}-A_{4}$ spermatogonia and subsequently for survival of type $B$ spermatogonia before they enter the meiotic stages of differentiation. A third recent report confirms the essential role played by c-kit in the initial stages of spermatogenesis (54). To study self-renewal and differentiation of spermatogonial stem cells the Authors transplanted undifferentiated testicular germ cells of the GFP transgenic mice into seminiferous tubules $S /$ or $W$ mutant mice. In the seminiferous tubules of $\mathrm{SI}$ mice, transplanted donor GFP expressing germ cells proliferated and formed colonies of undifferentiated $c$-kit negative spermatogonia but were unable to differentiate further. However, these undifferentiated but proliferating spermatogonia, retransplanted into SCF expressing seminiferous tubules of $W$ mutants, resumed differentiation, indicating that the transplanted donor germ cells contained spermatogonial stem cells and that stimulation of c-kit receptor by its ligand was necessary for maintenance of differentiated c-kit expressing type A spermatogonia but not for proliferation of undifferentiated ckit negative spermatogonial stem cells (54).

\section{POTENTIAL ROLE AT FERTILIZATION OF AN ALTERNATIVE C-KIT GENE PRODUCT EXPRESSED DURING SPERMIOGENESIS}

C-kit expression is down-regulated in coincidence with the onset of meiosis, whereas a a truncated $c$-kit product, tr-kit is specifically expressed in postmeiotic stages of spermatogenesis, due to the activity of a cryptic transcriptional promoter present within the $16^{\text {th }}$ intron of the c-kit gene $(18,22,27)$. This promoter drives haploid-specific expression of a reporter gene in transgenic mice $(22,55)$. Tr-kit is a $\sim 30 \mathrm{kDa}$ cytoplasmic protein, lacking the c-kit extra- 
cellular domain, the transmembrane domain, the first box of the split kinase domain (i.e. the ATP binding site) and the intervening sequence (including the PI3K docking site) of the intracellular domain, whereas it contains only the second box of the split kinase domain (i.e. the phosphotransferase catalytic site) and the carboxyterminal tail of the full-length c-kit receptor (Fig. 1). Tr-kit is accumulated in the residual sperm cytoplasm of the midpiece and in the postacrosomal region of mature spermatozoa, in proximity of the region involved in sperm-egg fusion during fertilization (56). Tr-kit causes parthenogenetic activation when microinjected into metaphase IIarrested oocytes (56). Tr-kit induced egg activation is mediated by activation of the $\gamma 1$ isoform of phospholipase C (PLC $\gamma 1)$, with consequent $\mathrm{IP}_{3}$-induced calcium mobilization from egg intracellular stores (57). Event hough tr-kit lacks the ATP-binding site of the $c$-kit tyrosine kinase domain, which is essential for $c$-kit catalytic function, tr-kit stimulates tyrosine phosphorylation of PLC $\gamma 1$ in transfected cell lines, suggesting that it interacts with a different tyrosine kinase present in the egg cytoplasm (57). The $\mathrm{SH} 3$ domain of PLC $\gamma 1$, but not the SH2 domains, is essential to mediate tr-kit-induced egg activation (57). However, mutation to phenylalanine of a tyrosine residue present in the tr-kit carboxyterminal region, which is a putative $\mathrm{SH} 2$-docking site, abolishes tr-kit-induced egg activation, suggesting that a $\mathrm{SH} 2$-containing adaptor protein intermediate is required to elicit $\mathrm{PLC} \gamma 1$ activation through the $\mathrm{PLC} \gamma 1 \mathrm{SH} 3$ domain (manuscript in preparation). Thus, this alternative $c^{-}$ kit gene product, which is specifically expressed in post-meiotic stages of spermatogenesis, and is present in mature spermatozoa, might play a role in the final function of the male gamete, which is to trigger early embryogenesis after sperm-egg fusion at fertilization (Fig. 2).

\section{ACKNOWLEDGMENTS}

This work was supported by CNR Targeted Project "Biotechnology" (R.G.), by CNR grant 98.00509.CT04 (P.R.), by grants from Ministero per l'Università e la Ricerca Scientifica e Tecnologica (P.R. and R.G.), and by Agenzia Spaziale Italiana.

\section{REFERENCES}

1. Besmer P., Murphy J.E., George P.C., Qiu F.H., Bergold P.J., Lederman L., Snyder H.W. Jr., Brodeur D., Zuckerman, E.E., Hardy W.D.

A new acute transforming feline retrovirus and relationship of its oncogene $v$-kit with the protein kinase gene family.

Nature 1986, 320: 415-421.
2. Yarden Y., Kuang W.J., Yang-Feng T., Coussens L., Munemitsu S., Dull T.J., Chen E., Schlessinger J., Francke U., Ullrich A.

Human proto-oncogene c-kit: a new cell surface receptor tyrosine kinase for an unidentified ligand. EMBO J. 1987, 6: 3341-3351.

3. Qiu F., Ray P., Barker P.E., Jhanwar S., Ruddle F.H., Besmer P.

Primary structure of c-kit: relationship with the CSF1/PDGF receptor kinase family - oncogenic activation of $\mathrm{v}$-kit involves deletion of extracellular domain and $\mathrm{C}$ terminus.

EMBO J. 1988, 7: 1003-1011.

4. Chabot B., Stephenson D.A., Chapman V.M., Besme P., Bernstein A.

The protooncogene c-kit encoding a transmembrane tyrosine kinase receptor maps to the mouse $\mathrm{W}$ locus. Nature 1988, 335: 88-89.

5. Geissler E.N., Ryan M.A., Housman D.E.

The dominant-white spotting $(W)$ locus of the mouse encodes the c-kit proto-oncogene. Cell 1988, 55: 185-192.

6. Russell E.S.

Hereditary anemias of the mouse: a review for geneticists.

Adv. Genet. 1979, 20: 357-459.

7. Witte O.N.

Steel locus defines new multipotent growth factor. Cell 1990, 63: 5-6.

8. Besmer P.

The kit ligand encoded at the murine steel locus: a pleiotropic growth and differentiation factor.

Curr. Opin. Cell. Biol. 1991, 3: 939-946.

9. Williams D.E., de Vries P., Namen A.E., Widmer M.B., Lyman S.D.

The Steel factor.

Dev. Biol. 1992, 151: 368-376.

10. Flanagan J.F., Chan D.C., Leder P.

Transmembrane form of the kit ligand growth factor is determined by alternative splicing and is missing in the Sld mutant.

Cell 1991, 64:1025-1035.

11. Matsui Y., Zsebo K.M., Hogan B.L.

Embryonic expression of a haematopoietic growth factor encoded by the SI locus and the ligand for c-kit. Nature 1990, 347: 667-669.

12. Dolci S., Williams D.E., Ernst M.K., Resnick J.L., Brannan C.I., Lock L.F., Lyman S.D., Boswell H.S., Donovan P.J.

Requirement for mast cell growth factor for primordial germ cell survival in culture.

Nature 1991, 352: 809-811.

13. Godin I., Deed R., Cooke J., Zsebo K., Dexter M., Wylie C.C.

Effects of the steel gene product on mouse primordial germ cells in culture.

Nature 1991, 352: 807-809. 
37. Mauduit C., Chatelain G., Magre S., Brun G., Benahmed M., Michel D.

Regulation by $\mathrm{pH}$ of the alternative splicing of the stem cell factor pre-mRNA in the testis.

J. Biol. Chem. 1999, 274: 770-775.

38. Koshimizu U., Sawada K., Tajima Y., Watanabe D., Nishimune $Y$.

White-spotting mutations affect the regenerative differentiation of testicular germ cells: demonstration by experimental cryptorchidism and its surgical reversal.

Biol. Reprod. 1991, 45: 642-648.

39. Brannan C.I., Bedell M.A., Resnick J.L., Eppig J.J., Handel M.A., Williams D.E., Lyman S.D., Donovan P.J., Jenkins N.A., Copeland N.G.

Developmental abnormalities in Steel17H mice result from a splicing defect in the steel factor cytoplasmic tail.

Genes Dev. 1992, 6: 1832-1842.

40. Hakovirta H, Yan W., Kaleva M., Zhang F., Vanttinen K., Morris P.L., Sode M., Parvinen. M., Toppari J. Function of stem cell factor as a survival factor of spermatogonia and localization of messenger ribonucleic acid in the rat seminiferous epithelium. Endocrinology 1999, 140: 1492-1498.

41. Tajima Y., Sawada K., Morimoto T., Nishimune Y. Switching of mouse spermatogonial proliferation from the c-kit receptor-independent type to the receptor-dependent type during differentiation.

J. Reprod. Fertil. 1994, 102: 117-122

42. Packer A.I., Besmer P., Bachvarova R.F.

Kit ligand mediates survival of type A spermatogonia and dividing spermatocytes in postnatal mouse testes. Mol. Reprod. Dev. 1995, 42: 303-310.

43. Yan W., Suominen J., Toppari J.

Stem cell factor protects germ cells from apoptosis in vitro. J. Cell Sci. 2000, 113: 161-168.

44. Allard E.K., Blanchard K.T., Boekelheide K. Exogenous stem cell factor (SCF) compensates for altered endogenous SCF expression in 2,5-hexanedione-induced testicular atrophy in rats.

Biol. Reprod. 1996, 55: 185-193.

45. Dirami G., Ravindranath N., Pursel V., Dym M.

Effects of stem cell factor and granulocyte macrophage-colony stimulating factor on survival of porcine type A spermatogonia cultured in KSOM. Biol. Reprod. 1999, 61: 225-230.

46. Dym M.

Spermatogonial stem cells of the testis.

Proc. Natl. Acad. Sci USA 1994, 91: 11287-11289.

47. Tajima Y., Sakamaki K., Watanabe D., Koshimizu U., Matsuzawa T., Nishimune Y.

Steel-Dickie (Sld) mutation affects both maintenance and differentiation of testicular germ cells in mice. J. Reprod. Fertil. 1991, 91: 441-449.
48. Timokhina I., Kissel H., Stella G., Besmer P. Kit signaling through PI 3-kinase and Src kinase pathways: an essential role for Rac1 and JNK activation in mast cell proliferation.

EMBO J. 1998, 17: 6250-6262.

49. De Sepulveda P., Okkenhaug K., Rose J.L., Hawley R.G., Dubreuil P., Rottapel R.

Socs 1 binds to multiple signalling proteins and suppresses steel factor-dependent proliferation. EMBO J. 1999, 18: 904-915.

50. Blume-Jensen P., Jiang G., Hyman R., Lee K.F., O'Gorman S., Hunter T.

Kit/stem cell factor receptor-induced activation of phosphatidylinositol $3^{\prime}$-kinase is essential for male fertility.

Nat. Genet. 2000, 24: 157-162.

51. Kissel H., Timokhina I., Hardy M.P., Rothschild G., Tajima Y., Soares V., Angeles M., Whitlow S.R., Manova K., Besmer P.

Point mutation in kit receptor tyrosine kinase reveals essential roles for kit signaling in spermatogenesis and oogenesis without affecting other kit responses.

EMBO J. 2000, 19: 1312-1326.

52. Serve H., Hsu Y.C., Besmer P.

Tyrosine residue 719 of the c-kit receptor is essential for binding of the P85 subunit of phosphatidylinositol (PI) 3-kinase and for c-kit-associated PI 3-kinase activity in COS-1 cells.

J. Biol. Chem. 1994, 269: 6026-6030.

53. Blume-Jensen P., Janknecht R., Hunter T.

The kit receptor promotes cell survival via activation

of PI 3-kinase and subsequent Akt-mediated phosphorylation of Bad on Ser136.

Curr. Biol. 1998, 8: 779-782.

54. Ohta H., Yomogida K., Dohmae K., Nishimune Y. Regulation of proliferation and differentiation in permatogonial stem cells: the role of c-kit and its ligand SCF.

Development 2000, 127: 2125-2131.

55. Herrmann B.G., Koschorz B., Wertz K., McLaughlin K.J., Kispert A.

A protein kinase encoded by the t complex responder gene causes non-mendelian inheritance. Nature 1999, 402: 141-146.

56. Sette C., Bevilacqua A., Bianchini A., Mangia F., Geremia R., Rossi P.

Parthenogenetic activation of mouse eggs by microinjection of a truncated c-kit tyrosine kinase present in spermatozoa.

Development 1997, 124: 2267-2274.

57. Sette C., Bevilacqua A., Geremia R., Rossi P. Involvement of phospholipase C 91 in mouse egg activation induced by a truncated form of the c-kit tyrosine kinase present in spermatozoa.

J. Cell Biol. 1998, 142: 1063-1074. 